\title{
Comparative photoelectrochemical studies of regioregular polyhexylthiophene with microdiamond, nanodiamond and hexagonal boron nitride hybrid films
}

Nunzio Giambrone ${ }^{1}$, Michael McCrory ${ }^{1}$, Ashok Kumar ${ }^{2}$ and Manoj K Ram ${ }^{3}$

1. Chemical and Biomedical Engineering Department, University of South Florida, Tampa, FL, USA

2. Mechanical Engineering Department, University of South Florida, Tampa, FL, USA

3. Clean Energy Research Center, University of South Florida, Tampa, FL, USA

nunzio@mail.usf.edu,kumar@usf.edu,mkram@usf.edu

\section{ABSTRACT}

Recently, we have studied photoelectrochemical properties of films of nanodiamond (ND)regioregular polyhexylthiophene (RRP3HT), ND-poly(3-dodecylthiophene), polyaniline, and other derivatives of polythiophene. The ND-RRP3HT, BN-RRP3HT and microdiamond (MD)RRPHTH films were characterized using ultraviolet-visible spectroscopy, Fourier transform infrared spectroscopy, scanning electron microscopy, Raman spectroscopy and x-ray diffraction techniques, respectively. The short circuit current, fill factor, and power conversion efficiency (PCE) for MD-RRP3HT, ND-RRP3HT and hexagonal boron nitride (hBN)-RRP3HT films were compared. The ND-RRP3HT nanostructured film revealed 8 to 20 times more photocurrent than most other nanostructured RRP3HT films in a photoelectrochemical cell. The ND-RRP3HT and hBN-RRP3HT films revealed improved efficiency compared to the MD- RRP3HT film. Due to the increased band gap, the hBN-RRP3HT have a higher PCE than ND as well as MD films. ND-RRP3HT and hBN-RRP3HT hybrid films exhibited comparable photoelectrochemical properties.

Keywords: hexagonal Boron Nitride, Nanodiamond, Microdiamond, Regioregular Polyhexylthiophene, Photovoltaic cell, Conducting Polymers 


\section{Introduction}

Organic/inorganic hybrid materials are gaining interest in the field of photoelectrochemical and photovoltaic cells in effort of increasing the power conversion efficiency (PCE) and lowering the cost [1-5]. Currently, 25.6\% is the highest PCE that has been achieved under solar radiation, using a crystalline silicon heterojunction under optimized short-circuit current $\left(\mathrm{J}_{\mathrm{SC}}\right)$ and optical losses $[6,7]$. Practically speaking, the high manufacturing cost does not allow practical use of photovoltaic cells based on silicon heterojunction technology [8]. The photoelectrochemical and photovoltaic properties of conducting polymers (polythiophene, polyethylenedioxythiophene, polyaniline, polyhexylthiophene, N-methyl thiophene, regioregular polyhexylthiophene (RRP3HT), etc.) containing metal oxide nanoparticles (zinc oxide, titanium oxide etc.) and quantum dots have been extensively studied [9-18]. The RRP3HT hybrid films have shown the great potential for higher PCE properties under light radiation [19-25].

Previously, photoelectrochemical properties of zinc oxide $(\mathrm{ZnO})$, titanium oxide $\left(\mathrm{TiO}_{2}\right)$ and nanodiamond (ND) with RRP3HT structures were investigated at length [26, 27]. The NDRRP3HT hybrid films showed 8 to 10 times superior photochemical properties compared to the ZnO- RRP3HT and $\mathrm{TiO}_{2}-\mathrm{RRP} 3 \mathrm{HT}$ hybrid structures [26]. The size of ND particles is varied for ND-RRP3HT hybrid film for photoelectrochemical studies. Recently, hexagonal boron nidtride $(\mathrm{hBN})$ and cubic boron nitride $(\mathrm{cBN})$ have both have gained interest in wear resistance, thermal shielding, chemical stability and lubricating applications [28, 30-32]. The pristine hBN, with a band gap of $5.5 \mathrm{eV}$ is highly insulating with $\mathrm{sp}^{2}$ configuration similar to graphite, can also be doped both as $\mathrm{p}$ or $\mathrm{n}$ type [28].. hBN has interesting structure where alternate boron and nitrogen are arranged in a honeycomb structure, and it shows the flexoelectric effect $[29,30]$. The hBN and NDs have been used in dielectric thermal fluids to enhance the thermal conductivity by 
dispersing the nanomaterial [31]. Besides, the nanofluid formation, hBN, ND and other nanomaterials are used in cooling electronics due to enhanced thermal properties [31]. The chemical vapor deposited $\mathrm{cBN}$ and nanocrystalline diamond composite films are used for enhanced mechanical properties of diamond [32]. The mechanical exfoliation of $\mathrm{cBN}$ is performed by deposition of a three layered cBN-ND film [33]. A nitrogen doped hBN monolayer on a nickel substrate can be used for catalytic reduction of oxygen, indicating that $\mathrm{hBN}$ is interesting material for thermal, catalytic and optical applications [34].

A spin coating machine is used to fabricate a thin hybrid film; which is a reliable method to reproduce the films for photoelectrochemical applications $[35,36]$. Our goal is to improve the power conversion efficiency of a single conjunction cell to translate the optical energy into electrical energy with a more sophisticated device using hBN-RRP3HT and ND-RRP3HT films.

\section{Experimental details}

\section{Materials:}

Various chemicals (chloroform, RRP3HT, propylene carbonate, tetraethylammonium tetrafluoroborate), hBN powder and microdiamond (MD) powder of $\sim 1 \mu \mathrm{m}$ in size, fluorine tin oxide (FTO) coated glass $(\sim 10 \Omega)$ were procured from Sigma-Aldrich. The ND particles size of 4 to $5 \mathrm{~nm}$ were bought from International Technology Center. The n-type silicon wafer (diameter 100+/-.05 mm, thickness 500-550 $\mu \mathrm{m}, 3000-4000 \Omega$ ) was procured from Monaco Silicon Technologies, Inc.

\section{Experimental procedure:}


Initially, $50 \mathrm{mg}$ of RRP3HT and $50 \mathrm{mg}$ of $\mathrm{ND}$, MD, or hBN was separately added in $50 \mathrm{ml}$ of chloroform, and stirred magnetically for 24 hours. The resulting solution containing RRP3HT with $\mathrm{ND}, \mathrm{MD}$, or hBN particles were used to fabricate films on FTO, quartz and silicon substrates, respectively. The substrates (n-type silicon wafers and FTO glass plates) were cleaned in acetone/methanol. Later, the substrates were wiped carefully with ethanol, rinsed in deionized water, and dried under nitrogen gas. The different layered hybrid films were fabricated on n-type silicon and FTO glass substrates using spin coating at a rotation of $600 \mathrm{rpm}$. The films were also annealed at $150{ }^{\circ} \mathrm{C}$ for an hour. Further, the films were left in air for 24 hours prior to various physical measurements.

\section{Results and discussions}

Ultraviolet-visible spectroscopy (UV-Vis) studies: The UV-vis measurements of various samples were measured using Jasco V-530 spectrophotometer. Figure 1 shows UV-vis spectra of the 5 layered hBN-RRP3HT, ND-RRP3HT, and MD- RRP3HT films on quartz substrates. Figure 1 curve 1 shows UV-vis absorption peaks at 523, 595 (small) and 973 (small) nm for the ND-RRP3HT film. The figure 1 curve 2 observes the peaks at 520 and 585 (small) nm for the ND-RRP3HT film. Figure 1 curve 3 reveals the peak at 401 (small) and $494 \mathrm{~nm}$ for the hBNRRP3HT film. The UV-vis peak at $520 \mathrm{~nm}$ is due to $\pi-\pi^{*}$ of conjugation in RRP3HT conducting polymer. There is a red shift at $494 \mathrm{~nm}$ due to the presence of hBN (curve 3) and blue shift at $523 \mathrm{~nm}$ (curve 2) due to presence of MD. There is an altered molecular arrangement of RR3HT blended with hBN than the ND film.

Fourier transform infrared spectroscopy (FTIR) studies: The infrared (IR) measurement on various samples were measured using FTIR spectrophotometer 'Spectrum One' from Perkin 
Elmer. FTIR spectra of the 5 layered hBN-RRP3HT, ND-RRP3HT, and MD-RRP3HT films on silicon substrate are shown in figure 2. Figure 2 (blue line) displays infrared peaks at 514, 567, $614,659,673,658,741,774,805,826,887,901,909,928,976,1050,1111,1384$, and $1609 \mathrm{~cm}^{-}$ ${ }^{1}$ for hBN-P3HT film of silicon substrates. Figure 2 (Green line) observes infrared peaks at 513, $565,511,655,681,740,784,815,828,878,907,966,1024,1058,1112,1440,1482,1498$, and $1658 \mathrm{~cm}^{-1}$ for ND-P3HT film of silicon substrates. Figure 2 (Orange line) shows the infrared peaks at 515, 569, 610, 739, and $1112 \mathrm{~cm}^{-1}$ for MD-P3HT film of silicon substrates.

X-ray diffraction (XRD) studies: XRD measurements of the hybrid films were measured using a Phillips Analytical XPert Pro Materials Research Diffractometer with Copper Ka1 X-ray source at $1.5406 \AA$. The XRD measurements were conducted using the grazing incident XRD (GIXRD) technique with the incident angle fixed at either $1.5^{\circ}(\mathrm{MD}$ and $\mathrm{BN})$ or $2^{\circ}(\mathrm{ND})$. X-ray diffraction patterns of the samples were studies to understand the crystallinity for $2 \theta$ angles ranging from 5 to 90 degrees of the MD-RRP3HT film is shown in figure 3 . The characteristic (111) and (220) orientation due to polycrystalline diamond is observed in Figure 3 [37]. It has sharp peak at around (551), and has also two narrow peaks at around $56^{\circ}$. The microcrystalline structure in blended film is not influenced due the presence of RRP3HT. Figure 3 (b) reveals hybrid structure for ND with RRP3HT film. The ND-RRP3HT film does not involve the surface chemical bonding and its crystallinity structure is not influenced. So, compressed (111) and (220) orientations in the ND are observed in RRP3HT film [38]. The X-ray diffraction studies of $\mathrm{hBN}-\mathrm{RRP} 3 \mathrm{HT}$ film reveals intense peak at $26^{\circ}$ and wide band at $45^{\circ}$ due to presence of $\mathrm{hBN}$ in the structure [39]. However, other characteristics peaks at $41^{\circ}$ and $55^{\circ}$ are suppressed due to the presence of conducting polymer, which affects crystallinity in the film. 
Scanning electron microscopy (SEM) studies: The morphology on the film was investigated using a Field Emission Hitachi S800 Scanning Electron Microscope with Energy Dispersive Spectroscopy capabilities. The morphology on the film was investigated using a Field Emission Hitachi S800 Scanning Electron Microscope with EDS capabilities operating at $25 \mathrm{kV}$. Figure 4 (a \& b) shows SEM structure of RRP3HT, hBN-RRP3HT, ND-RRP3HT, and MD-RRP3HT films deposited on silicon substrates. Figure 4a shows regular RRP3HT film on silicon substrates. The MD-RRP3HT films appears as a regular RRP3HT film with MD particles in the structure. Figure $4 \mathrm{~b}$ illustrates that MD does not make a homogenous hybrid structure, and there is clear separation of MD particles with RRP3HT, whereas ND is well dispersed in ND-RRP3HT hybrid films as shown in Figure 4 (a \& b). The hBN-RRP3HT film reveals the aggregation of a few hBN particles within RRP3HT, however, it also shows completely different structure than either RRP3HT or ND- RRP3HT films as shown in figure 4.

Electrochemical studies: The electrochemical measurements of the samples were performed using model "PGZ 301 Dynamic EIS voltammetry" from Voltalab in two electrodes configurations where the film deposited on FTO glass plate worked as working electrode and platinum as a counter electrode in an electrolyte containing $0.1 \mathrm{M}$ tetraethylammonium tetrafluoroborate (TEATFB) in acetonitrile. The CVs were studied at different scan rate (5 $\mathrm{mV} / \mathrm{sec}$ to $100 \mathrm{mV} / \mathrm{sec})$. Figure $5(\mathrm{a}, \mathrm{b}, \mathrm{c})$ shows the cyclic voltammetry $(\mathrm{CV})$ curves of 2 layers of MD- RRP3HT, ND-RRP3HT and hBN - RRP3HT on FTO glass in 0.1M TEATFB based electrolyte. The MD- RRP3HT displays the redox potential peak at $0.5 \mathrm{~V}$ (curve 1) which has been shifted to $0.4 \mathrm{~V}$ (curve 2) due to light exposure as shown in figure 4a. The enhancement of 
CV of MD-RRP3HT hysteresis shows an increase of current at $1 \mathrm{~V}$ due light radiation from a 60 watt light bulb. Figure 4b observes CV of ND-RRP3HT with and without light exposure. The redox peak at $0.34 \mathrm{~V}$ has been shifted to $0.38 \mathrm{~V}$, besides an appearance of new peak at $0.74 \mathrm{~V}$ and with light exposure the current density increases to $55 \mu \mathrm{A} / \mathrm{cm}^{2}$. Figure $4 \mathrm{c}$ shows CV of 2layered $\mathrm{hBN}-\mathrm{RRP3HT}$ with and without light exposure. The $\mathrm{CV}$ shows distinct peaks at $0.23 \mathrm{~V}$ and $0.52 \mathrm{~V}$, which has been shifted to $0.30 \mathrm{~V}$ and $0.55 \mathrm{~V}$. However, the $\mathrm{CV}$ of hBN - RRP3HT shows better capacitive value in positive potential from 0 to $1 \mathrm{~V}$.

Figure 6 shows the CV of 5 Layers of either hBN-RRP3HT, ND-RRP3HT, or MD-RRP3HT on FTO glass substrates from $1.5 \mathrm{~V}$ to $-1.5 \mathrm{~V}$. A greater current density is reached with the $\mathrm{hBN}-$ RRP3HT film due to lower band gap and strain energy. In comparison to the micron-sized diamonds, the nano-sized diamonds provide a higher photocurrent. However, the expected photocurrent has been observed in the reverse biasing of current.

The chronoamperometric study of the films deposited on FTO glass plate worked as working electrode and platinum as a counter electrode in an electrolyte containing $0.1 \mathrm{M}$ tetraethylammonium tetrafluoroborate (TEATFB) in acetonitrile. The chronoamperometric study was performed with application of different potential from $(1000 \mathrm{mV}$ to $100 \mathrm{mV})$. However, Figure 7 shows chronoamperometric study at $-1000 \mathrm{mV}$ for ND-RRP3HT (curve 1) and hBNRRP3HT (curve 2) for 5-layered film in propylene carbonate containing TEATFB. Inset in figure 7 is the chronoamperometric curve of the MD-RRP3HT film. Interestingly, switching on and off the light source develops a larger photocurrent for the hBN-RRP3HT than the ND-RRP3HT film, while the MD-RRP3HT film develops a smaller current. This is most likely due to the fact 
that the MD does not act as electron accepter, but actually forms an insulating layer and the photocurrent is simply due to the RRP3HT film.

The photocurrent study of the hBN-RRP3HT, ND-RRP3HT, or MD-RRP3HT film deposited on FTO glass plate worked as working electrode and platinum as a counter electrode in an electrolyte containing $0.1 \mathrm{M}$ tetraethylammonium tetrafluoroborate (TEATFB) in acetonitrile from -1 to $1 \mathrm{~V}$. Figure 8 shows the photocurrent of hBN-RRP3HT, ND-RRP3HT, and MDRRP3HT films due to light exposure. The hBN-RRP3HT reveals higher photocurrent than both ND-RRP3HT and MD-RRP3HT films for potential window from -1 to $1 \mathrm{~V}$. The photocurrent increases as a function applied potential. However, the straight lines fitting of the photocurrent as function of potential has been shown for MD- RRPHTH, ND-RRP3HT and BN-RRP3HT based electrodes.

The possible band diagram has been shown in Figure 9 for the RRP3HT, MD- RRPHTH, NDRRP3HT and BN-RRP3HT structures.

PCE $(\eta)$, fill factor $(\mathrm{FF})$ and with maximum power $\left(\mathrm{P}_{\mathrm{MAX}}\right)$ were calculated using the data from the CV curves. The fill factor (FF) was calculated by the quotient of a cell's maximum power to the voltage at open current $\left(\mathrm{V}_{\mathrm{oc}}\right)$ and the short circuit current $\left(\mathrm{I}_{\mathrm{sc}}\right)$. $\mathrm{P}_{\mathrm{MAX}}$ was estimated as a product of $\mathrm{V}_{\mathrm{oc}}, \mathrm{I}_{\mathrm{sc}}$, and FF estimated using eq.1 to 3.. The $\eta$ was estimated for each cell by dividing $\mathrm{P}_{\text {MAX }}$ by the amount of power taken in by cell. In this case amount of 1-Sun illumination was used.

$$
\begin{aligned}
& F F=\frac{V_{M P} I_{M P}}{V_{O C} I_{S C}} \\
& V_{O C}=V_{O C} I_{S C} F F
\end{aligned}
$$




$$
\eta=\frac{V_{O C} I_{S C} F F}{P_{\text {in }}}
$$

As predicted, the $\eta$ of the hBN-RRP3HT (0.12) film was higher than those calculated for the ND-RRP3HT (0.056), and MD-RRP3HT (0.045) films. The hBN-RRP3HT film has shown an increase in the efficiency over both ND-RRP3HT and MD-RRP3HT films. The increase in efficiency in hBN-RRP3HT is due to an increase in the photocurrent of the cell.

\section{Conclusion}

The photoelectrochemical behavior of hBN-RRP3HT, ND-RRP3HT, and MD-RRP3HT films has been studied. The hBN-RRP3HT films were found to have better photoelectrochemical properties than ND-RRP3HT films. The hBN has a lower band gap donor along with less strain energy than diamond, and the band gap donor levels have been found directly to correlate with PCE in a positive linear relationship. The photoelectrochemical devices produced using hBNRRP3HT and ND-RRP3HT films have advantages over solid photovoltaic based devices due to the easy formation of solid/liquid junctions, direct photo energy conversion, not sensitive to defect in the blended structures, and the production cost is minimal. The external voltage of hBN-RRP3HT and ND-RRP3HT films can be varied with and without the reference electrode to attain the photocurrent. Due to large band gap of $>5.5 \mathrm{eV}$ of $\mathrm{hBN}$, it has negative electron affinity. However, its unique layered structure, which is similar to graphene, has not been investigated in detail and we are trying to understand the phenomena for future work. The hBN with AiN:Mg has shown 6 to 7 orders of magnitude less photocurrent, which could be happening due to the nanohybrid formation with RRPHTh [40]. However, the nano -size variation of hBN will be studied in future for infusion of $\mathrm{hBN}$ on the nanoparticle level. 
Acknowledgments This work was supported by the National Science Foundation with award number 1066643.

\section{Figures captions}

Figure 1: UV-Visible spectra of (curve 1) MD- RRPHTH, (curve 2) ND-RRP3HT and (curve 3) BN-RRP3HT films on quartz

Figure 2: FTIR spectra of (curve 1) MD- RRPHTH, (curve 2), ND-RRP3HT and (curve 3) BNRRP3HT films on silicon substrate

Figure 3: X-ray diffraction patterns of MD- RRP3HT, ND-RRP3HT and BN-RRP3HT of 5-layer blends films.

Figure 4 SEM images of 5-layered RRP3HT, MD- RRPHTH, ND-RRP3HT and BN-RRP3HT at two magnifications

Figure 5: Cyclic voltammogram of 2-layered MD- RRPHTH, (b) ND-RRP3HT and (c) BNRRP3HT films on FTO glass substrate in $\mathrm{LiClO}_{4}$ and propylene carbonate electrolyte

Figure 6: Cyclic voltammogram of 5-layered MD- RRPHTH, (b) ND-RRP3HT and (c) BNRRP3HT films on FTO glass substrate in $\mathrm{LiClO}_{4}$ and propylene carbonate electrolyte (the light was signed for longer period and effect of heating)

Figure 7: Chronoamperometric study of 5-layered ND-RRP3HT (curve 1) and BN-RRP3HT (curve 2) films in $\mathrm{LiClO}_{4}$ and propylene carbonate electrolyte at $-1000 \mathrm{mV}$ and inset is the NDRRP3HT

Figure 8: The light exposure effect on the current for MD- RRPHTH (curve 1), ND-RRP3HT (curve 2) and BN-RRP3HT (curve 3) films.

Figure 9: Band diagram of photoelectrochemical cell based on RRP3HT, MD- RRPHTH, NDRRP3HT and BN-RRP3HT structure 


\section{References}

[1] H. Zhou, Q. Chen, G. Li, S. Luo, T.-b. Song, H.-S. Duan, Z. Hong, J. You, Y. Liu, Y. Yang, Interface engineering of highly efficient perovskite solar cells, Science, 345 (2014) 542-546.

[2] N. Li, D. Baran, G.D. Spyropoulos, H. Zhang, S. Berny, M. Turbiez, T. Ameri, F.C. Krebs, C.J. Brabec, Environmentally printing efficient organic tandem solar cells with high fill factors: a guideline towards $20 \%$ power conversion efficiency, Advanced Energy Materials, 4 (2014).

[3] S.P. Lim, A. Pandikumar, Y.S. Lim, N.M. Huang, H.N. Lim, In-situ electrochemically deposited polypyrrole nanoparticles incorporated reduced graphene oxide as an efficient counter electrode for platinum-free dye-sensitized solar cells, Scientific reports, 4 (2014).

[4] Y. Yu, J. Li, D. Geng, J. Wang, L. Zhang, T.L. Andrew, M.S. Arnold, X. Wang, Development of Lead Iodide Perovskite Solar Cells Using Three-Dimensional Titanium Dioxide Nanowire Architectures, ACS nano, DOI (2014).

[5] S. Mathew, A. Yella, P. Gao, R. Humphry-Baker, B.F. Curchod, N. Ashari-Astani, I. Tavernelli, U. Rothlisberger, M.K. Nazeeruddin, M. Grätzel, Dye-sensitized solar cells with $13 \%$ efficiency achieved through the molecular engineering of porphyrin sensitizers, Nature chemistry, 6 (2014) 242-247.

[6] B. Demaurex, J.P. Seif, S. Smit, B. Macco, W.E. Kessels, J. Geissbuhler, S. De Wolf, C. Ballif, Atomic-Layer-Deposited Transparent Electrodes for Silicon Heterojunction Solar Cells, DOI (2014).

[7] K. Masuko, M. Shigematsu, T. Hashiguchi, D. Fujishima, M. Kai, N. Yoshimura, T. Yamaguchi, Y. Ichihashi, T. Mishima, N. Matsubara, Achievement of more than 25\% conversion efficiency with crystalline silicon heterojunction solar cell, DOI (2014).

[8] A. Chen, K. Zhu, Computer simulation of a-Si/c-Si heterojunction solar cell with high conversion efficiency, Solar Energy, 86 (2012) 393-397.

[9] W.J. Beek, M.M. Wienk, R.A. Janssen, Efficient hybrid solar cells from zinc oxide nanoparticles and a conjugated polymer, Advanced Materials, 16 (2004) 1009-1013.

[10] M. Grätzel, Photoelectrochemical cells, Nature, 414 (2001) 338-344.

[11] P.V. Kamat, Quantum dot solar cells. Semiconductor nanocrystals as light harvesters $\dagger$, The Journal of Physical Chemistry C, 112 (2008) 18737-18753.

[12] Y. Hao, M. Yang, W. Li, X. Qiao, L. Zhang, S. Cai, A photoelectrochemical solar cell based on $\mathrm{ZnO} / \mathrm{dye}$ /polypyrrole film electrode as photoanode, Solar energy materials and solar cells, 60 (2000) 349-359.

[13] H. Li, J. Li, Q. Xu, X. Hu, Poly (3-hexylthiophene)/TiO2 nanoparticle-functionalized electrodes for visible light and low potential photoelectrochemical sensing of organophosphorus pesticide chlopyrifos, Analytical chemistry, 83 (2011) 9681-9686.

[14] Y. Hao, J. Pei, Y. Wei, Y. Cao, S. Jiao, F. Zhu, J. Li, D. Xu, Efficient semiconductorsensitized solar cells based on poly (3-hexylthiophene)@ CdSe@ ZnO core- shell nanorod arrays, The Journal of Physical Chemistry C, 114 (2010) 8622-8625.

[15] J. Boucle, S. Chyla, M.S. Shaffer, J.R. Durrant, D.D. Bradley, J. Nelson, Hybrid Solar Cells from a Blend of Poly (3-hexylthiophene) and Ligand-Capped TiO2 Nanorods, Advanced functional materials, 18 (2008) 622-633.

[16] J.H. Heo, S.H. Im, H.-j. Kim, P.P. Boix, S.J. Lee, S.I. Seok, I.n. Mora-Seró, J. Bisquert, Sb2S3-sensitized photoelectrochemical cells: open circuit voltage enhancement through 
the introduction of poly-3-hexylthiophene interlayer, The Journal of Physical Chemistry C, 116 (2012) 20717-20721.

[17] P.A. Basnayaka, P. Villalba, M.K. Ram, L. Stefanakos, A. Kumar, Photovoltaic properties of multi walled carbon nanotubes-poly (3-octathiophene) conducting polymer blends structures, MRS Proceedings, Cambridge Univ Press, 2013, pp. 139-144.

[18] F. Alvi, M.K. Ram, H. Gomez, R.K. Joshi, A. Kumar, Evaluating the chemio-physio properties of novel zinc oxide-polyaniline nanocomposite polymer films, Polymer journal, 42 (2010) 935-940.

[19] M.T. Dang, L. Hirsch, G. Wantz, P3HT: PCBM, best seller in polymer photovoltaic research, Advanced Materials, 23 (2011) 3597-3602.

[20] W.U. Huynh, J.J. Dittmer, N. Teclemariam, D.J. Milliron, A.P. Alivisatos, K.W. Barnham, Charge transport in hybrid nanorod-polymer composite photovoltaic cells, Physical Review B, 67 (2003) 115326.

[21] A. Goetzberger, C. Hebling, H.-W. Schock, Photovoltaic materials, history, status and outlook, Materials Science and Engineering: R: Reports, 40 (2003) 1-46.

[22] P. Peumans, S. Uchida, S.R. Forrest, Efficient bulk heterojunction photovoltaic cells using small-molecular-weight organic thin films, Nature, 425 (2003) 158-162.

[23] D. Wei, G. Amaratunga, Photoelectrochemical cell and its applications in optoelectronics, Int. J. Electrochem. Sci, 2 (2007) 897-912.

[24] Y. Chen, Z. Jiang, M. Gao, S.E. Watkins, P. Lu, H. Wang, X. Chen, Efficiency enhancement for bulk heterojunction photovoltaic cells via incorporation of alcohol soluble conjugated polymer interlayer, Applied Physics Letters, 100 (2012) 203304.

[25] S. Berson, R. de Bettignies, S. Bailly, S. Guillerez, B. Jousselme, Elaboration of P3HT/CNT/PCBM composites for organic photovoltaic cells, Advanced Functional Materials, 17 (2007) 3363-3370.

[26] M.K. Ram, H. Gomez, F. Alvi, E. Stefanakos, Y. Goswami, A. Kumar, Novel Nanohybrid Structured Regioregular Polyhexylthiophene Blend Films for Photoelectrochemical Energy Applications, The Journal of Physical Chemistry C, 115 (2011) 21987-21995.

[27] C. Zhuang, L. Wang, Z. Dai, D. Yang, High Frequency Ni-NiO-Ag Metal-InsulatorMetal Tunnel Diodes Fabricated via Anodic Aluminum Oxide Templates, ECS Solid State Letters, 4 (2015) P39-P42.

[28] R.Y. Tay, M.H. Griep, G. Mallick, S.H. Tsang, R.S. Singh, T. Tumlin, E.H.T. Teo, S.P. Karna, Growth of large single-crystalline two-dimensional boron nitride hexagons on electropolished copper, Nano letters, 14 (2014) 839-846.

[29] K.K. Kim, A. Hsu, X. Jia, S.M. Kim, Y. Shi, M. Dresselhaus, T. Palacios, J. Kong, Synthesis and characterization of hexagonal boron nitride film as a dielectric layer for graphene devices, ACS nano, 6 (2012) 8583-8590.

[30] I. Naumov, A.M. Bratkovsky, V. Ranjan, Unusual Flexoelectric Effect in TwoDimensional Noncentrosymmetric s p 2-Bonded Crystals, Physical review letters, 102 (2009) 217601.

[31] S.S. Sedarous, C.D. Attlesey, Nanofluids for use in cooling electronics, US Patents, US20120186789, 2012.

[32] Y.M. Chong, K.L. Ma, K.M. Leung, C.Y. Chan, Q. Ye, I. Bello, W. Zhang, S.T. Lee, Synthesis and mechanical properties of cubic boron nitride/nanodiamond composite films, Chemical Vapor Deposition, 12 (2006) 33-38. 
[33] H. Li, K. Leung, W. Zhang, Thick and adherent (cBN/nano-diamond) 3 multilayer films deposited by rf magnetron sputtering, Sixth International Conference on Thin Film Physics and Applications, International Society for Optics and Photonics, 2008, pp. 69842S-69842S-69844.

[34] A. Lyalin, A. Nakayama, K. Uosaki, T. Taketsugu, Functionalization of monolayer h-BN by a metal support for the oxygen reduction reaction, The Journal of Physical Chemistry C, 117 (2013) 21359-21370.

[35] D.B. Mitzi, L.L. Kosbar, C.E. Murray, M. Copel, A. Afzali, High-mobility ultrathin semiconducting films prepared by spin coating, Nature, 428 (2004) 299-303.

[36] C. Lawrence, The mechanics of spin coating of polymer films, Physics of Fluids (19581988), 31 (1988) 2786-2795.

[37] O. Auciello, A.V. Sumant, Status review of the science and technology of ultrananocrystalline diamond ( $\mathrm{UNCD}^{\mathrm{TM}}$ ) films and application to multifunctional devices, Diamond and related materials, 19 (2010) 699-718.

[38] W.-W. Zheng, Y.-H. Hsieh, Y.-C. Chiu, S.-J. Cai, C.-L. Cheng, C. Chen, Organic functionalization of ultradispersed nanodiamond: synthesis and applications, Journal of Materials Chemistry, 19 (2009) 8432-8441.

[39] L.M.S. Ansaloni, E.M.B. de Sousa, Boron Nitride Nanostructured: Synthesis, Characterization and Potential Use in Cosmetics, DOI (2013).

[40] R. Dahal, J. Li, S. Majety, B. Pantha, X. Cao, J. Lin, H. Jiang, Epitaxially grown semiconducting hexagonal boron nitride as a deep ultraviolet photonic material, Applied Physics Letters, 98 (2011) 211110. 


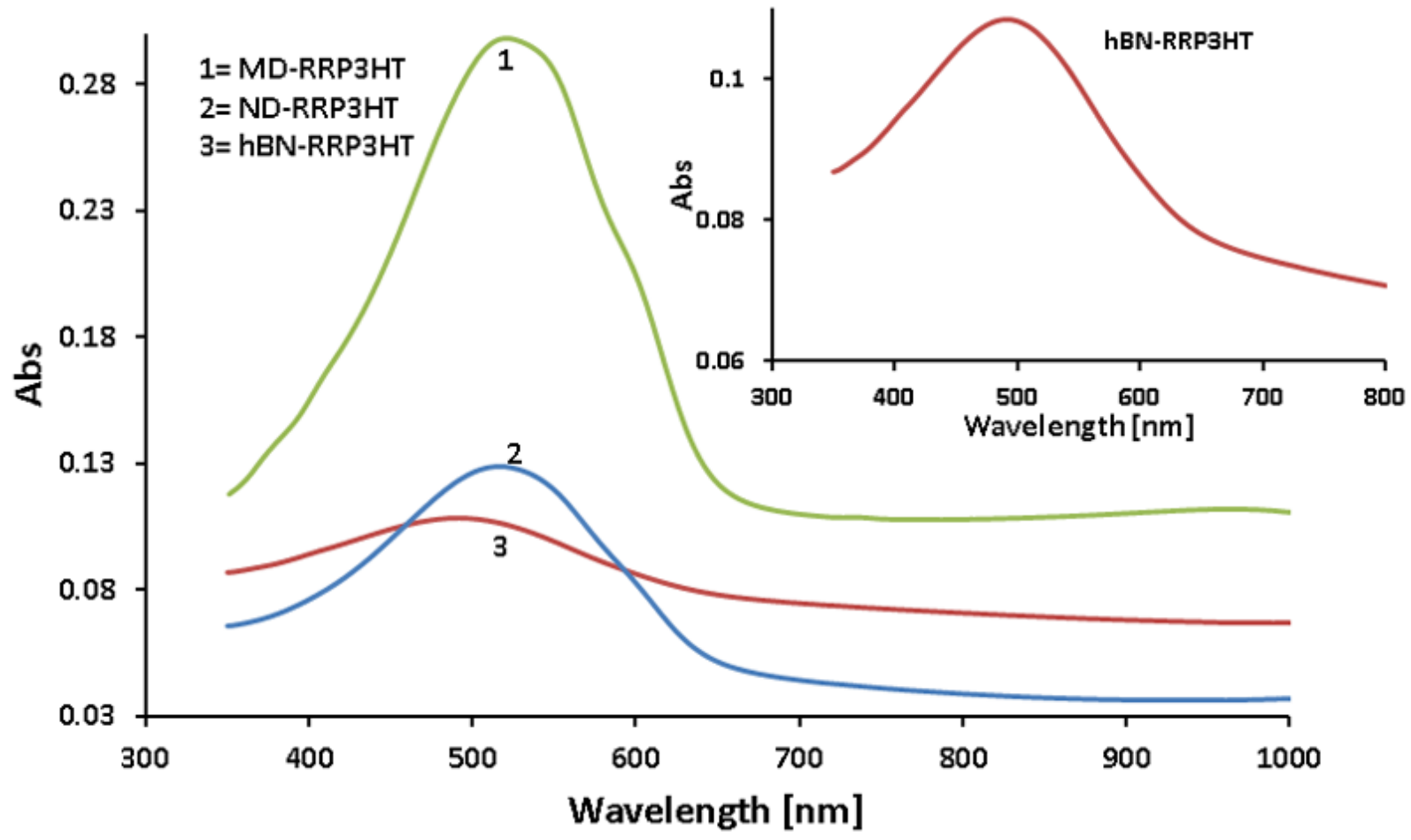

FIGURES 
Figures 2

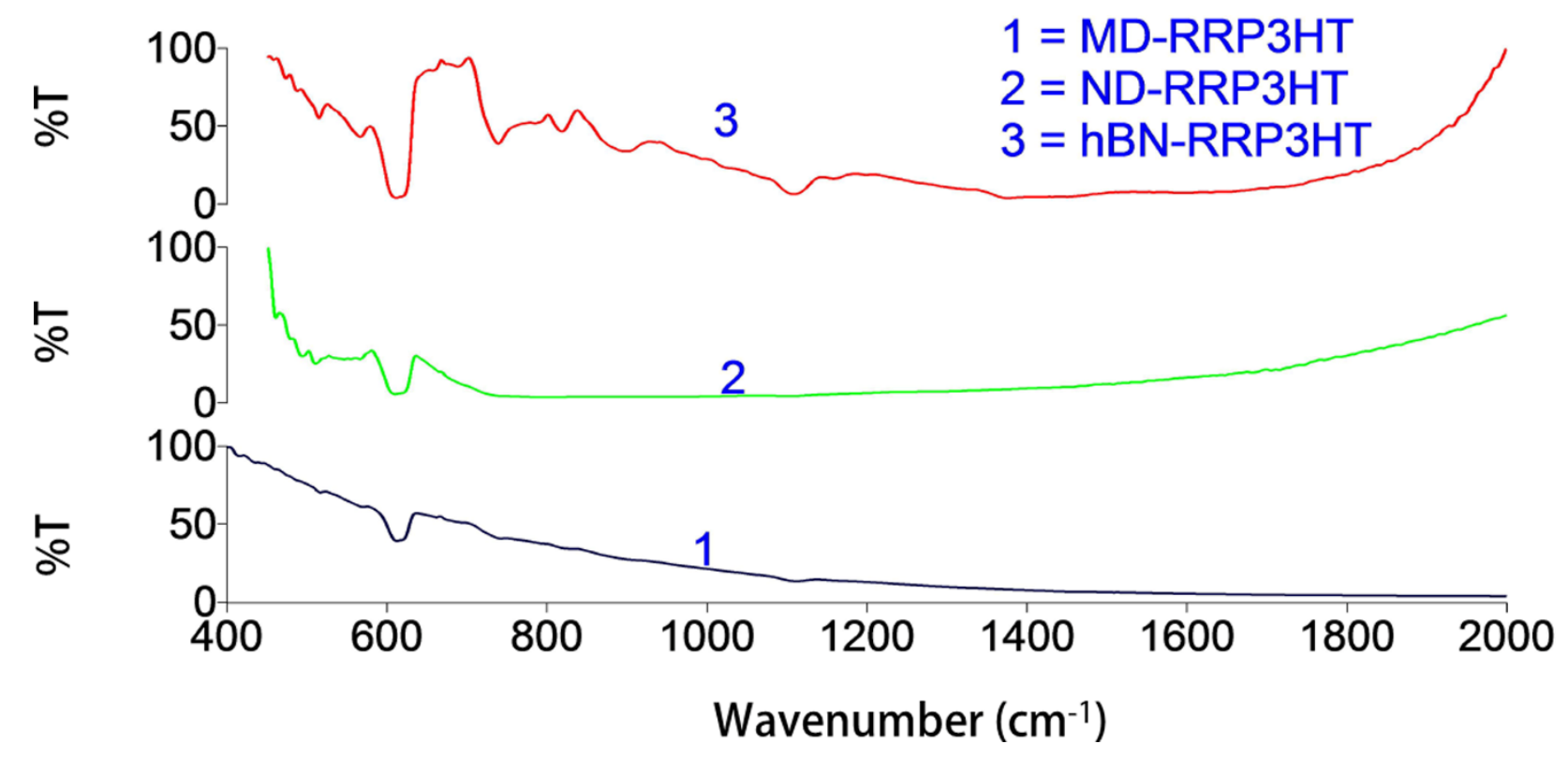



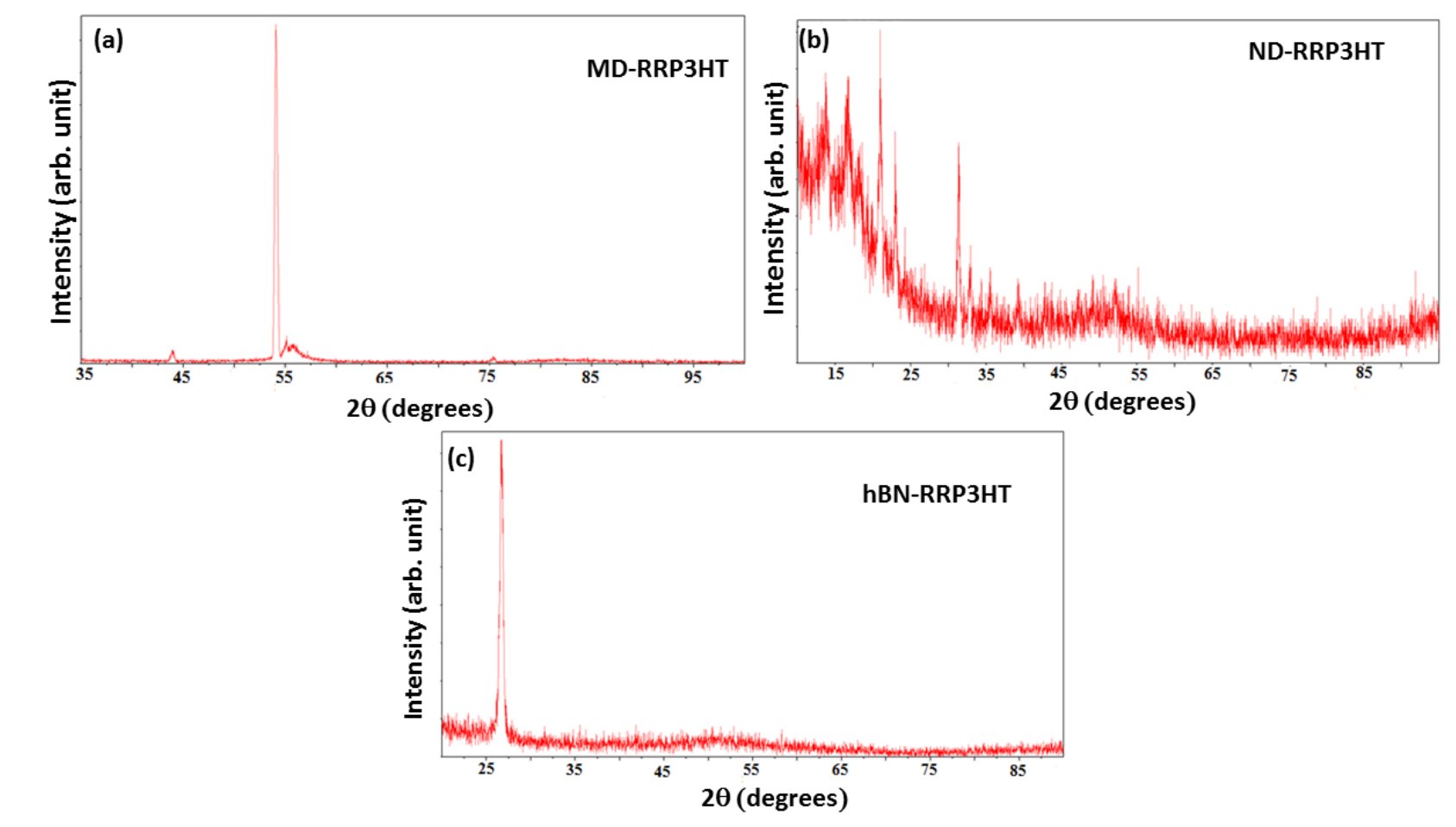

\section{Figures 3}




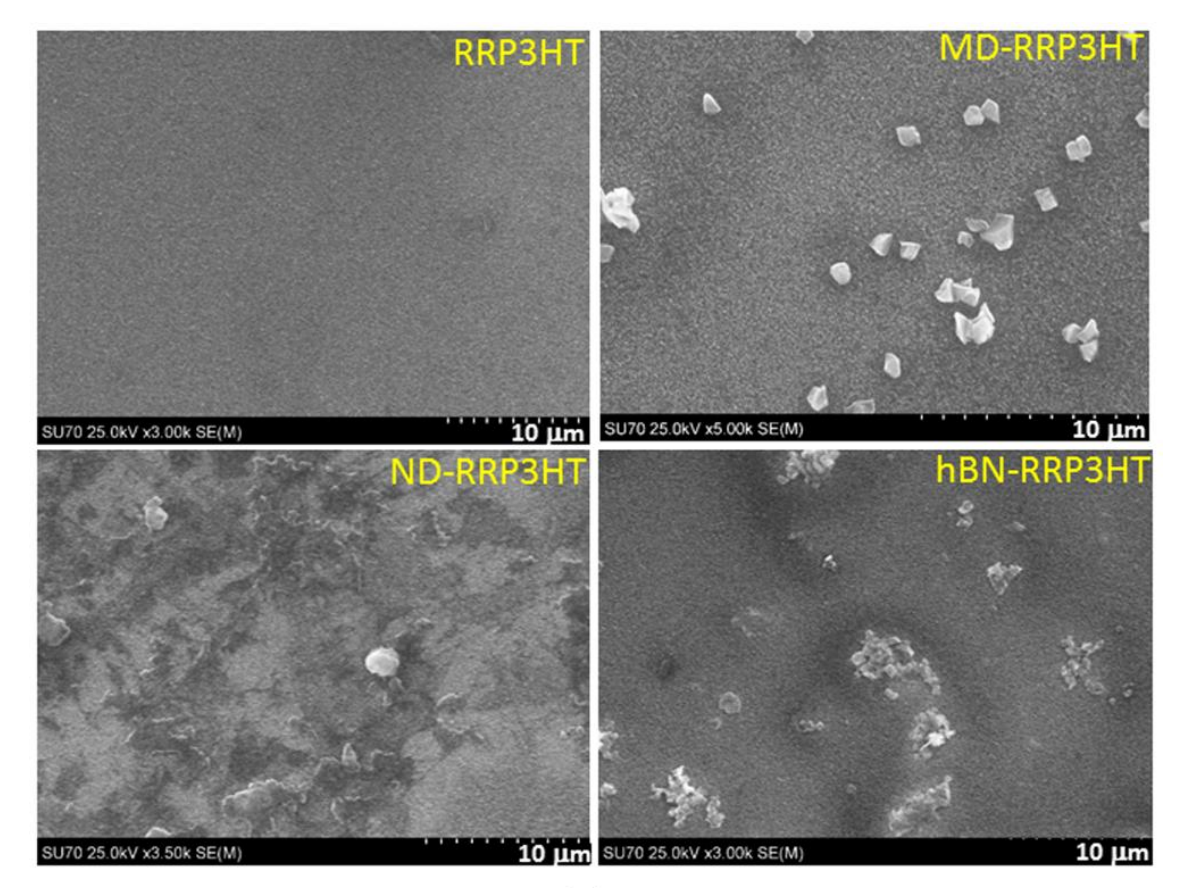

(a)
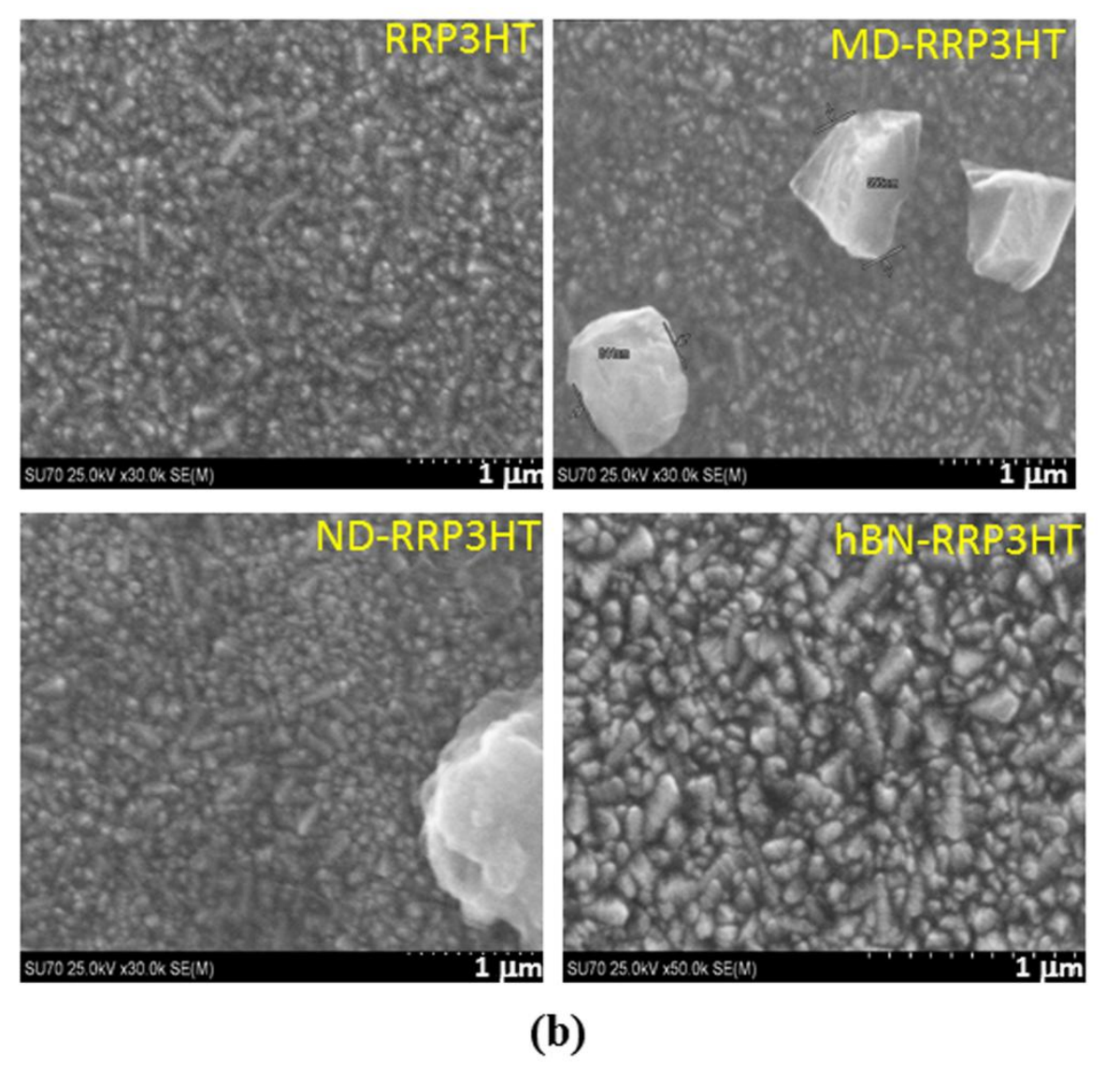

(b)

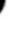

.

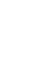
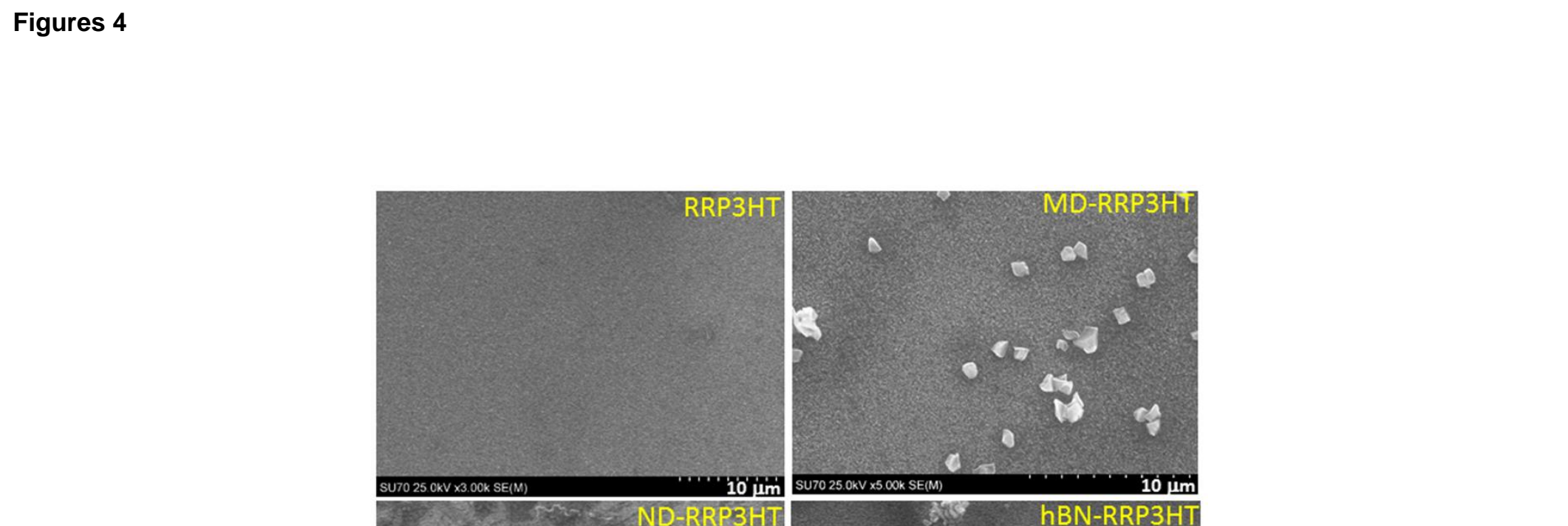

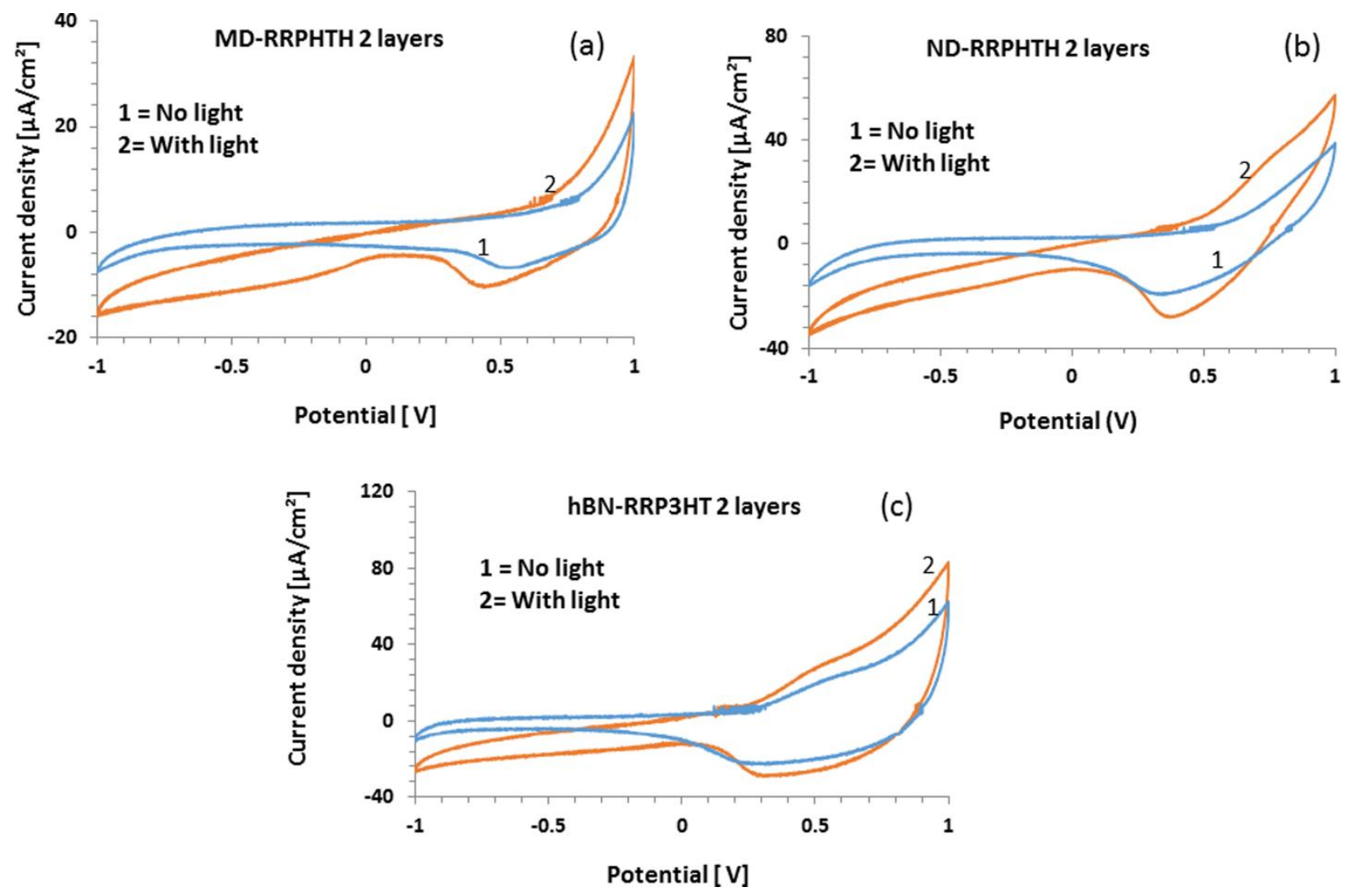


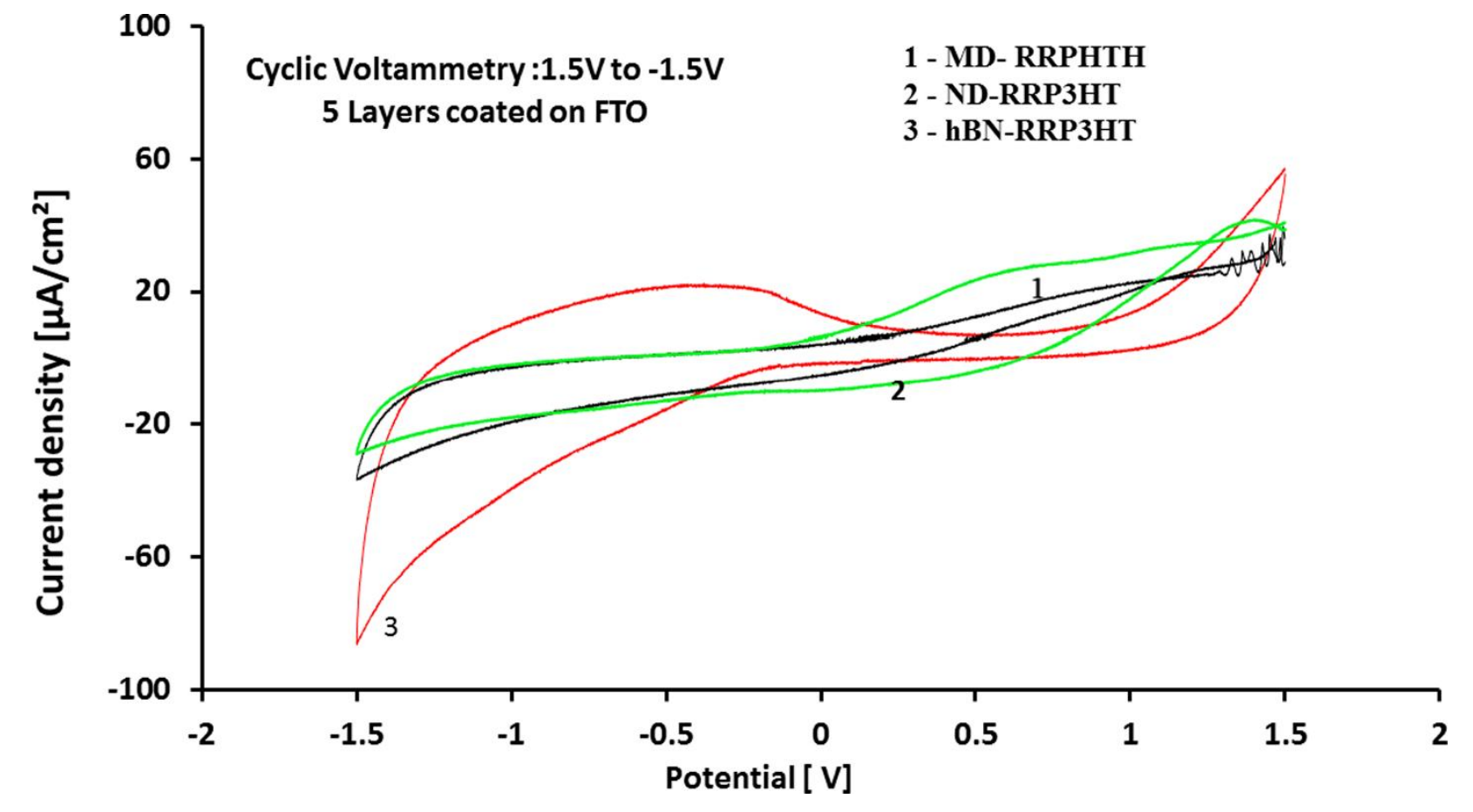

Figures 6 


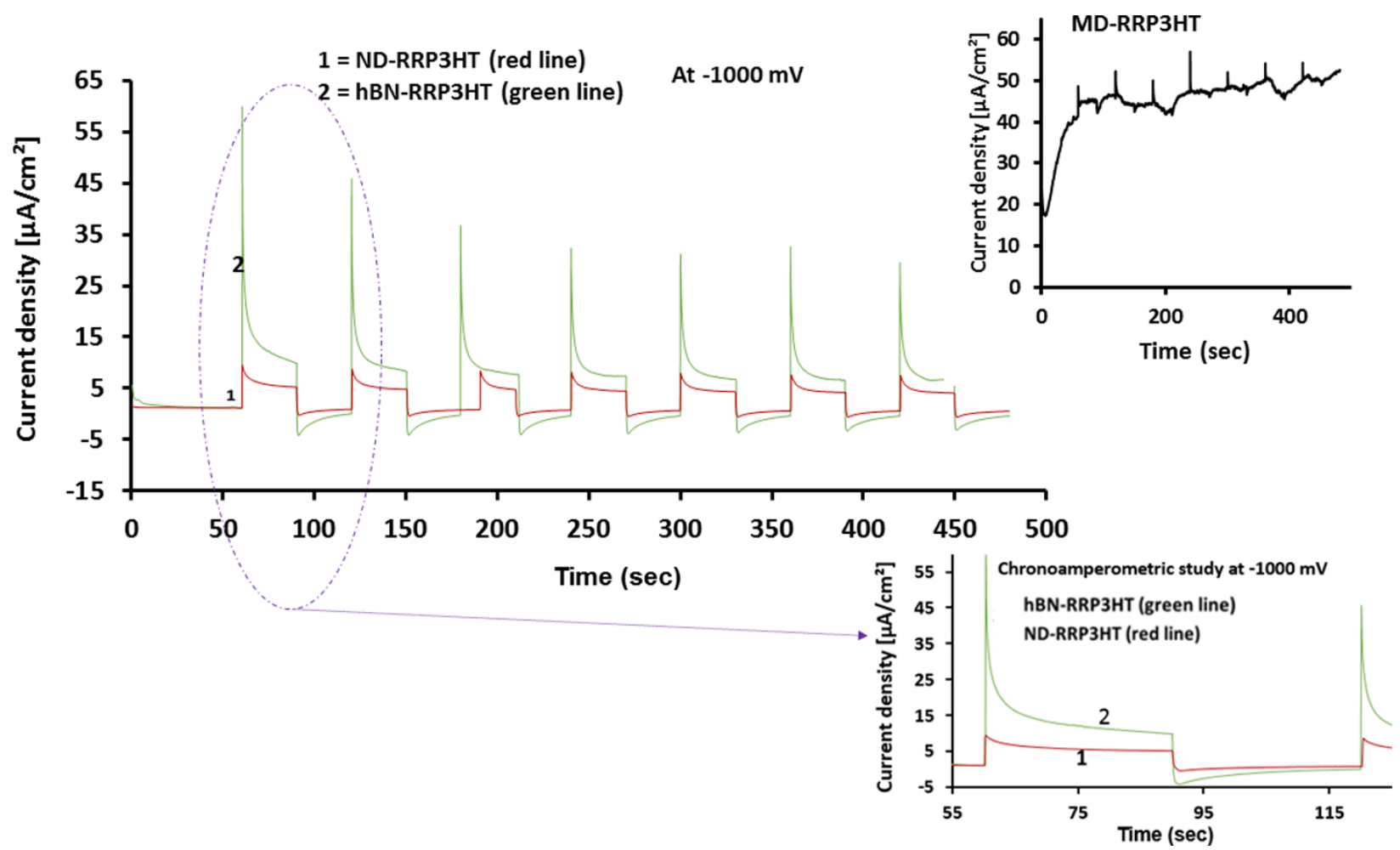




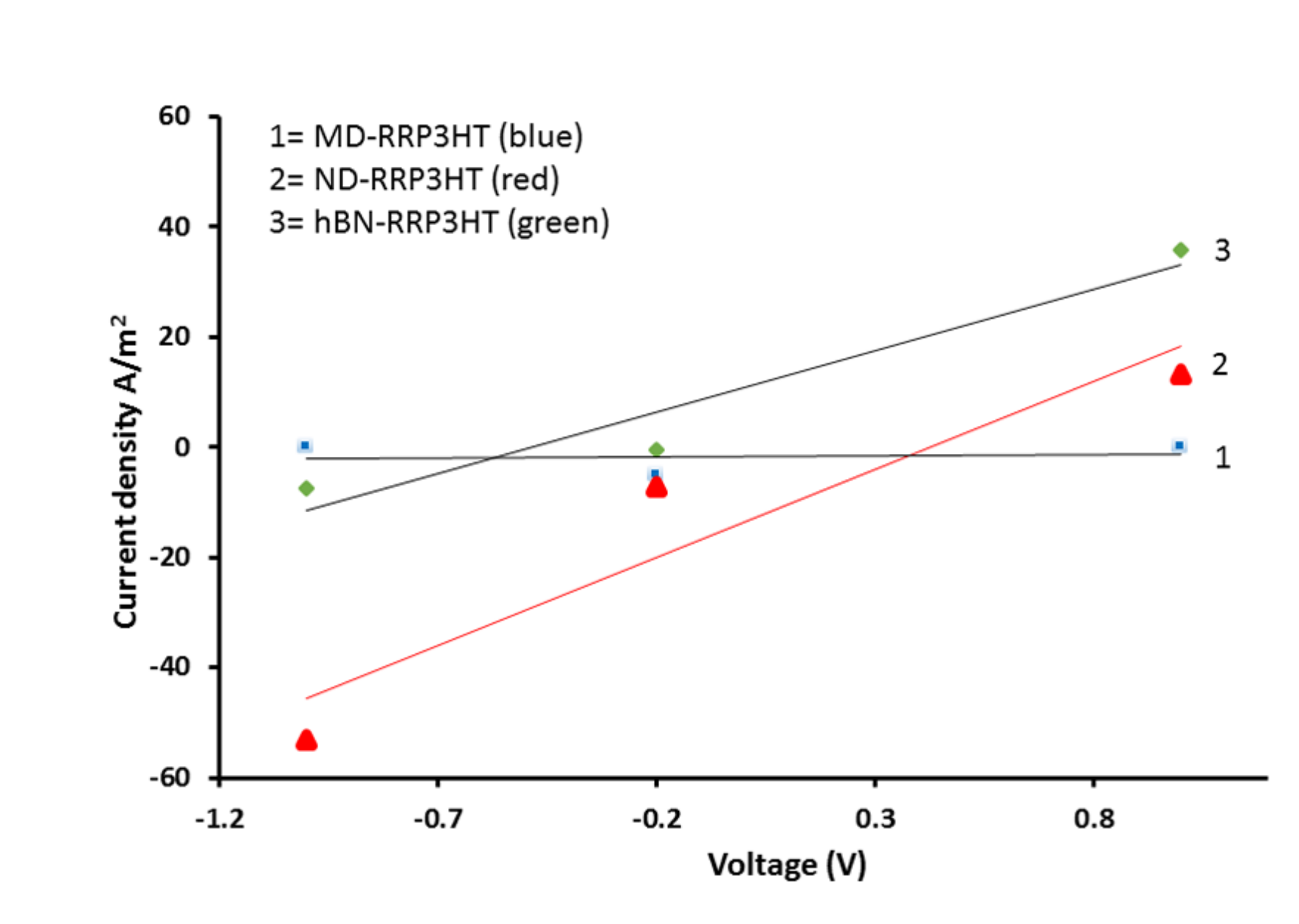

Figures 8

Fures 8

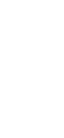




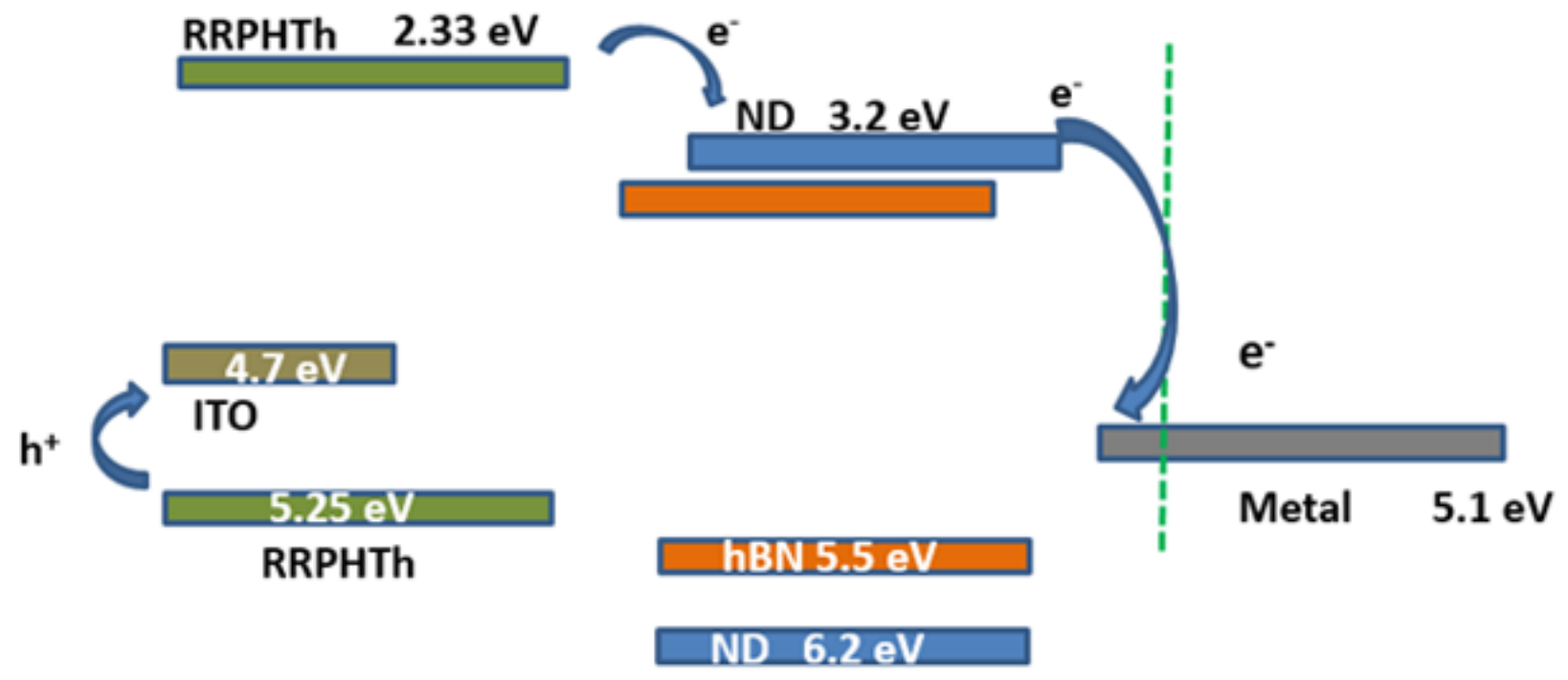

